\title{
QUILLEN'S WORK ON THE FOUNDATIONS OF CYCLIC COHOMOLOGY
}

\author{
JOACHIM CUNTZ
}

\begin{abstract}
We give a survey of Quillen's contributions, apart from the very first result in [19, to the area of cyclic homology.
\end{abstract}

Dedicated to the memory of Daniel Quillen

\section{INTRODUCTION}

Daniel Quillen had been interested in cyclic homology from the very start. After the first paper on the connection between cyclic homology and the Lie algebra homology of matrices over an algebra [19], his papers aimed at a deep understanding of the fundamental structures underlying cyclic theory. When I went through his articles on the subject I was again impressed by the systematic development of his thoughts on the subject and by the very thorough analysis of every detail which never left any loose ends.

A starting point for his work on the subject was the search for a more conceptual explanation for Connes' construction of cyclic cocycles from algebra extensions. He did this in [27], 25] using an elegant formalism involving noncommutative versions of Chern character forms and of Chern-Simon forms. He also showed that the important JLO-cocycle [16] could be obtained from a similar construction. In [27], he showed that all cocycles for an algebra $A$ can be obtained from an extension of the form $I \longmapsto R \rightarrow A$ using traces on $R / I^{n}$ or on $I^{n}$. In this way he could represent the cyclic cohomology groups $H C^{2 n} A$ and $H C^{2 n+1} A$, as inductive limits, over all extensions of $A$, of the spaces of traces on $R / I^{n+1}$ or on $I^{n+1}$, respectively.

At about the same time, A.Connes and the author had studied traces on the ideal $\varepsilon A$ in a universal so-called semi-split extension $\varepsilon A \longmapsto E A \rightarrow A$ and had shown that one obtains the cyclic cohomology from traces on powers of that ideal (or quotients by these powers) [4. It was noted in [6] that there is a bijection between traces on powers of $\varepsilon A$ and traces on powers of the ideal in a free extension of $A$. This observation gave another proof of Quillen's result in [27] and was the beginning of a long lasting collaboration of Dan Quillen and the author. This collaboration culminated in [11] and in a new approach to cyclic homology (especially well adapted to the periodic theory) and in a proof of excision in periodic cyclic theory in [13].

Date: August 16, 2021.

2000 Mathematics Subject Classification. Primary: 22D25, 46L89, 11R04, 11M55.

Key words and phrases. cyclic homology, quasifree algebra, $X$-complex, periodic cyclic theory, excision.

Research supported by DFG through CRC 878 and by ERC through AdG 267079. 
Some of the concepts developed along the way such as in particular the one of smoothness (quasi-freeness) in noncommutative geometry also became influential. A basis for the approach to cyclic homology developed in [11] is a very simple - nearly trivial - periodic complex of period 2, the $X$-complex $X(A)$ which was already used by Quillen in [26]. It is a reduction of the cyclic bicomplex (or equivalently of the $B, b$-bicomplex) to the lowest dimensions 0 and 1 . Even though it is so simple it is still good enough to capture the cyclic (and Hochschild) homology for algebras of homological dimension $\leq 1$. Since, in particular, free algebras have that property, such algebras were called quasi-free in [10]. The properties of quasifree algebras were analyzed in [10]. Interestingly they turn out to be exactly the natural generalization of the notion of a smooth variety or algebra, to the non-commutative setting. In fact, they are characterized by a lifting property which is the exact analogue of the corresponding property of a smooth algebra in the commutative category.

For an arbitrary algebra $A$ the cyclic homology/cohomology can then be obtained in the following way. Choose any extension of $A$ of the form $0 \rightarrow I \rightarrow R \rightarrow A \rightarrow 0$ with $R$ quasifree and consider the $I$-adic completion $\hat{R}=\underset{\longleftarrow}{\lim } R / I^{n}$.

Then the periodic cyclic homology $H P_{*}(A)$ is simply the homology of the complex $X_{*}(\hat{R})$, the periodic cyclic cohomology $H P^{*}(A)$ is the homology of the (continuous for the $I$-adic topology) dual of $X_{*}(A)$ and the ordinary cyclic homology/cohomology groups $H C_{n}(A), H C^{n}(A)$ can be obtained from a natural filtration (a small modification of the $I$-adic filtration) of these complexes. This procedure is directly analogous to the construction of infinitesimal homology in algebraic geometry where one embeds a general variety into a smooth variety, completes and the considers the de Rham complex of the completion. The $X$-complex thus plays the role of a noncommutative de Rham complex.

The simplicity of this description of cyclic theory is a little bit obscured in [11] by the fact that the exposition there strives to elucidate all facets of the approach as well as its connections to other approaches.

The concepts involved in this approach also were a natural basis for an attack on the problem of excision for periodic cyclic homology/cohomology. The excision problem for the ordinary cyclic theory had been understood for quite a while. Wodzicki [32] had shown that excision does not always hold and that it holds, given an algebra $I$, for any extension of the form $I \longmapsto A \rightarrow A / I$ if and only if $I$ has a property which he called $H$-unitality. On the other hand, Goodwillie [14] had shown that excision in the periodic cyclic theory also holds for nilpotent ideals (which are never $H$-unital). It was therefore natural to ask whether excision holds for arbitrary extensions in the periodic cyclic theory. That problem had remained open for a long time. In [9] it was discovered that every ideal in a quasi-free algebra satisfies a property which was called approximate $H$-unitality and that this property is enough to prove excision. Since any algebra can be represented as a quotient $R / I$ of a quasi-free (even free) algebra and since the periodic cyclic cohomology of $A$ can be easily related to that of $I$ in such an extension, excision in periodic cyclic cohomology followed in complete generality. 
The proof of excision for periodic homology (rather than cohomology) and for the bivariant case needed some additional ideas and techniques. It fits naturally into the framework of the $X$-complex and quasi-free extensions, and was developed in [13. The proof in that paper takes a detour by reducing the general problem first to the quasi-free case and then applying an argument à la Wodzicki. This detour was avoided in a later simplified proof due to R.Meyer [20], which fits perfectly into the framework of [11].

In this note we give a brief introduction to some of the main ideas in the series of 10 articles that have been written in the period between 1987 and 1997. The reader will notice the linear progression of Quillen's thoughts on the subject. Several of the results from that period represent fundamental new findings. The notion of noncommutative smoothness (being quasi-free) that has been analyzed in much detail in [10], has become influential in various contexts, see e.g. [18]. The new description of cyclic theory in [11] has become the framework of choice for topological theories such as entire and local cyclic theory, cf. [21], [24], but also for equivariant cyclic theory, [31], 30]. Finally, the excision result [13] has opened the way to a better understanding of cyclic theory, to the construction of a bivariant Chern character on suitable categories of algebras, [7] [24] and has been generalized to other cyclic theories such as the entire and the local theory.

\section{Cyclic homology And Algebra extensions}

Let $I \longmapsto R \rightarrow A$ be an extension (i.e. a short exact sequence where the arrows are algebra homomorphisms) of the algebra $A$. Note that, in this article, every algebra is an algebra over a field of characteristic 0, which we usually even assume to be the field of complex numbers $\mathbb{C}$. To a trace (i.e. a linear functional vanishing on commutators) on $R / I^{n}$ or on $I^{n+1}$, Connes [5] had associated cyclic cocycles in $H C^{2 n}$ and $H C^{2 n+1}$, respectively. Dually, Connes' construction leads to natural maps

$$
H C_{2 n}(A) \rightarrow R /\left(I^{n+1}+[R, R]\right) \quad H C_{2 n+1}(A) \rightarrow I^{n+1} /\left[I, I^{n}\right]
$$

where $[\cdot, \cdot \cdot$ denotes the linear space generated by all commutators. In [26], Quillen, introducing an elegant formalism, showed how to interpret the cyclic cocycles obtained using the dual maps from the spaces of traces on $R / I^{n}$ or on $I^{n+1}$ to cyclic cohomology, as Chern character maps and Chern-Simons forms. He also showed that for large $n$ they are related by Connes' $S$-operator. For these considerations he used already formulas that came to be important later in [11] and in particular he already used the $X$-complex in order to prove the $S$-relations. His construction of cyclic cocycles using the formalism of Chern and Chern-Simons forms has been used later by various authors in connection with index theorems, see e.g. [22], [15].

In [27], Quillen then showed that the maps in (1) are injective, if $R$ is a free algebra. Thus, every cyclic cocycle for $A$ can be represented by a trace on $R / I^{n}$ or on $I^{n}$, if $R$ is a free extension of $A$. From this he then obtains the following description of cyclic homology 


$$
H C_{2 n}=\lim _{\longleftarrow}\left(R /\left(I^{n+1}+[R, R]\right)\right) \quad H C_{2 n+1}=\lim _{\longleftarrow}\left(I^{n+1} /\left[I, I^{n}\right]\right)
$$

where the inverse limit is taken over all extensions of $A$ of the form $0 \rightarrow I \rightarrow R \rightarrow$ $A \rightarrow 0$.

On the other hand, at about the same time when Quillen was working on [27], Connes and the author had analyzed traces on the free product algebra $Q A=A \star A$ and had shown that they are described by families of multilinear functionals that describe cocycles in the cyclic bicomplex [4]. It was noted in [6] that the canonical free extension $R A$ of an algebra $A$ given by the tensor algebra over $A$ is a natural subalgebra of $Q A$ (the even part for a natural $\mathbb{Z} / 2$-grading) and that therefore, traces on $R A$ correspond bijectively to graded traces on $Q A$. This observation gave an alternative proof for Quillen's results in [27] and was the starting point for a long lasting cooperation between the author and Dan Quillen.

At the end of the introduction to the paper [27], Quillen writes: "It is clear from the present paper, with its extensive use of explicit complexes and formulas, that a true Grothendieck understanding of cyclic cohomology remains a goal for the future. Indeed, the inverse limit formula for cyclic homology described above is a strong indication that there is a much simpler foundation of the subject."

The search for such a formulation was the guideline in Quillen's subsequent work and in our collaboration.

\section{OpERATORS ON DIFFERENTIAL FORMS AND CONVENIENT DESCRIPTION OF THE CYCLIC BICOMPLEX}

We will have to refer to one of the standard definitions of cyclic homology. The most convenient way to describe the fundamental cyclic bicomplex for our purposes is in the guise of the $B, b$-bicomplex defined by operators $B$ and $b$ on the algebra $\Omega A$ of differential forms over a given algebra $A$. We go immediately in medias res and introduce the important harmonic decomposition of $\Omega A$ which has been developed in [12] and used in [11] by Cuntz-Quillen.

Given an algebra $A$, we denote by $\Omega A$ the universal algebra generated by $x \in A$ with relations of $A$ and symbols $d x, x \in A$, where $d x$ is linear in $x$ and satisfies $d(x y)=x d(y)+d(x) y$. We do not impose $d 1=0$, i.e., if $A$ has a unit, $d 1 \neq 0 . \Omega A$ is a direct sum of subspaces $\Omega^{n} A$ generated by linear combinations of $x_{0} d x_{1} \ldots d x_{n}$, and $d x_{1} \ldots d x_{n}, x_{j} \in A$. This decomposition makes $\Omega A$ into a graded algebra. We write $\operatorname{deg}(\omega)=n$ if $\omega \in \Omega^{n} A$.

As a vector space, for $n \geq 1$,

$$
\Omega^{n} A \cong \widetilde{A} \otimes A^{\otimes n} \cong A^{\otimes(n+1)} \oplus A^{\otimes n}
$$

(where $\widetilde{A}$ is $A$ with a unit adjoined, and $1 \otimes x_{1} \otimes \cdots \otimes x_{n}$ corresponds to $d x_{1} \ldots d x_{n}$ ). The operator $d$ is defined on $\Omega A$ by

$$
d\left(x_{0} d x_{1} \ldots d x_{n}\right)=d x_{0} d x_{1} \ldots d x_{n} \quad d\left(d x_{1} \ldots d x_{n}\right)=0
$$


The operator $b$ is defined by

$$
b(\omega d x)=(-1)^{\operatorname{deg} \omega}[\omega, x] \quad b(d x)=0, b(x)=0, \quad x \in A, \omega \in \Omega A
$$

Then clearly $d^{2}=0$ and one easily computes that also $b^{2}=0$. Under the isomorphism in equation (2) $d$ becomes

$$
d\left(x_{0} \otimes \ldots \otimes x_{n}\right)=1 \otimes x_{0} \otimes \ldots \otimes x_{n} \quad d\left(1 \otimes x_{1} \otimes \ldots \otimes x_{n}\right)=0
$$

while $b$ corresponds to the usual Hochschild operator $A^{\otimes(n+1)} \rightarrow A^{\otimes n}$. Another important natural operator is the degree (or number) operator defined by $N(\omega)=$ $\operatorname{deg}(\omega) \omega$. Now, for the operator $L=(N d) b+b(N d)$ one obtains a splitting $\Omega A=$ Ker $L \oplus \operatorname{Im} L$ (this follows from a polynomial identity satisfied by the Karoubi operator $\kappa=d b+b d)$.

The operator $L$ thus behaves like a "selfadjoint" operator. It can be viewed as an abstract Laplace operator on the algebra of abstract differential forms $\Omega A$. We denote by $P$ the projection onto the kernel of $L$. The elements in the image of $P$ are then "abstract harmonic forms", [12], [11].

By construction, $P$ commutes with $b, d, N$. Thus setting $B=N P d$ one finds $B b+b B=P L=0$ and $B^{2}=0$.

The preceding identities show that we obtain a bicomplex - the $(B, b)$-bicomplex in the following way

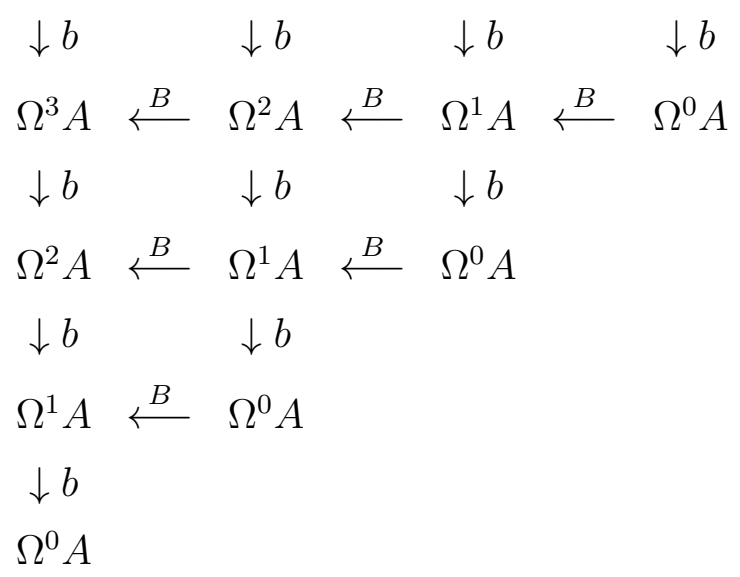

One can rewrite the $(B, b)$-bicomplex (3) using the isomorphism $\Omega^{n} A \cong A^{\otimes(n+1)} \oplus$ $A^{\otimes n}$ in equation (2). An easy computation shows that then it becomes the usual cyclic bicomplex with the operators $b, b^{\prime}$ and Connes' signed cyclic permutation operator $\lambda$.

Definition 3.1. One defines the cyclic homology $H C_{n}(A)$ of the algebra $A$ to be the homology of the total complex of the $B$,b-bicomplex.

We denote by $\widehat{\Omega} A$ the infinite product

$$
\widehat{\Omega} A=\prod_{n} \Omega^{n} A
$$


and by $\widehat{\Omega}^{e v} A, \widehat{\Omega}^{\text {odd }} A$ its even and odd part, respectively. $\widehat{\Omega} A$ may be viewed as the (periodic) total complex for the bicomplex (3) continued infinitely to the left and down. Similarly, the (continuous for the filtration topology) dual $(\widehat{\Omega} A)^{\prime}$ of $\widehat{\Omega} A$ is

$$
(\widehat{\Omega} A)^{\prime}=\bigoplus_{n}\left(\Omega^{n} A\right)^{\prime}
$$

Definition 3.2. The periodic cyclic homology $H P_{*}(A), *=0,1$, is defined as the homology of the $\mathbb{Z} / 2$-graded complex

$$
\widehat{\Omega}^{e v} A \underset{B+b}{\stackrel{B+b}{\rightleftarrows}} \widehat{\Omega}^{\text {odd } A}
$$

and the periodic cyclic cohomology $H P^{*}(A), *=0,1$, is defined as the homology of the $\mathbb{Z} / 2$-graded complex

$$
\left(\widehat{\Omega}^{e v} A\right)^{\prime} \quad \underset{B+b}{\stackrel{B+b}{\leftrightarrows}}\left(\widehat{\Omega}^{o d d} A\right)^{\prime}
$$

The comparison of the $B, b$-bicomplex with the $X$-complex for a quasi-free resolution suggests in fact the boundary operator $B-b$ rather than $B+b$.. This convention would also avoid complicated signs in the Chern character map. We follow here however the conventions in [11].

\section{The $X$-COMPleX And QUASI-FreE AlgeBras}

The $X$-complex is the quotient of the $B, b$-bicomplex (3) by the following subbicomplex

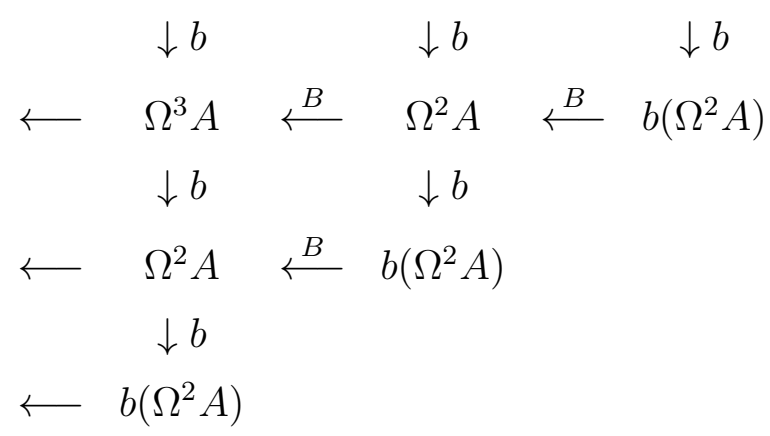

Thus $X(A)$ is the periodic complex

$$
X(A): \quad \rightarrow A \stackrel{\natural d}{\longrightarrow} \Omega^{1} A_{\natural} \stackrel{b}{\longrightarrow} A \stackrel{\natural d}{\longrightarrow} \Omega^{1} A_{\natural} \rightarrow
$$

where $\Omega^{1} A_{\natural}=\Omega^{1} A /\left[A, \Omega^{1} A\right]$ is the quotient of the bimodule $\Omega^{1} A$ by the subspace of commutators and $\downarrow: \Omega^{1} A \rightarrow \Omega^{1} A_{\natural}$ is the canonical quotient map.

Even though the $X$-complex is very simple, it is good enough to compute the periodic cyclic theory for a special class of algebras - the quasi-free algebras for which the subcomplex by which we divide is contractible.

Proposition-Definition 4.1. ([29], [10]) Let $A$ be an algebra. The following conditions are equivalent: 
(1) Let $0 \rightarrow N \rightarrow S \stackrel{q}{\rightarrow} B \rightarrow 0$ be an extension of algebras where the ideal $N$ is nilpotent (i.e., $N^{k}=\{0\}$ for some $k \geq 1$ ) and $A \stackrel{\alpha}{\longrightarrow} B$ a homomorphism. Then there exists a homomorphism $A \stackrel{\alpha^{\prime}}{\longrightarrow}$ S such that $q \circ \alpha^{\prime}=\alpha$.

(2) A has cohomological dimension $\leq 1$ with respect to Hochschild cohomology. The algebra $A$ is called quasi-free if these equivalent conditions are satisfied.

Many other different characterizations of quasi-freeness are analyzed in [10]. An especially important property for us is the fact that $H X_{*}(A)=H P_{*} A$ for a quasifree algebra $A$ (where $H X_{*}(A), *=0,1$ denotes the homology of the complex $X(A)$ ).

The fact that $H P_{*}$ is invariant under polynomial (or differentiable) homotopies, is reflected by the fact that the $X$-complex has, for quasifree algebras a homotopy invariance property which is described by a natural Cartan homotopy formula.

The most important examples of quasi-free algebras are free algebras. In particular the non-unital tensor algebra $T A$ given by

$$
T A=A \oplus A^{\otimes^{2}} \oplus A^{\otimes^{3}} \oplus \ldots
$$

is quasi-free and the natural quotient map $T A \rightarrow A$ defines a quasi-free extension of $A$.

Another interesting feature of quasi-free algebras, which justifies to consider quasifreeness as the correct analogue of smoothness in the noncommutative situation, is the following "tubular neighbourhood theorem" proved in [10].

Theorem 4.2. [10] [6, Theorem 2]. Assume $A$ is quasi-free. Consider an extension $A=R / I$ and let $N$ denote the A-bimodule $I / I^{2}$. If $A$ and $R$ are quasi-free, there is an isomorphism $u: \hat{T}_{A} N \rightarrow \hat{R}$ from the $N$-adic completion $\hat{T}_{A} N$ of the tensor algebra $T_{A} N$ to the $I$-adic completion of $R$ which extends the identity map on $A \oplus N$.

The concept of smoothness for a noncommutative algebra studied in [10], was later found to be great significance in noncommutative geometry, cf. e.g. [18].

\section{CyClic homology AND NONSINGUlarity}

Let $p: A \rightarrow T A$ be the canonical linear inclusion of $A$ into the tensor algebra over $A$ (see (5) above) and, for $x, y \in A$ set $\omega(x, y)=p(x y)-p x p y$. Then the map

$$
\alpha: x_{0} d x_{1} d x_{2} \ldots d x_{2 n-1} d x_{x_{2 n}} \mapsto x_{0} \omega\left(x_{1}, x_{2}\right) \ldots \omega\left(x_{2 n-1}, x_{2 n}\right)
$$

defines a linear isomorphism $\Omega^{e v} A \stackrel{\alpha}{\longrightarrow} T A$. In fact, this isomorphism becomes an algebra isomorphism for a deformation of the product on $\Omega^{e v} A$, the Fedosov product. It extends to a linear isomorphism $\alpha: \Omega A \rightarrow X(T A)$ respecting the $\mathbb{Z} / 2$-grading. May be the most important theorem in [11] which nearly seems like a miracle is the following.

Theorem 5.1. [11] The map a gives an isomorphism (not just a quasi-isomorphism!) between the $\mathbb{Z} / 2$-graded complexes $P \Omega A$ (with boundary operator $B+b$ ) and $P X(T A)$ which respects a natural filtration on both sides. 
Here $P$ denotes the harmonic projection operator, see section 3 , on $\Omega A$ and also its counterpart on $X(T A)$. Due to the definition and properties of $P$, the complements $P^{\perp} \Omega A$ and $P^{\perp} X(T A)$ do not have any homology. As a consequence all cyclic invariants such as cyclic homology, cyclic cohomology, periodic cyclic theory, bivariant cyclic theory can be defined and computed alternatively from the $B, b$-bicomplex or from the complex $X(T A)$.

Thus one can define all cyclic homology/cohomology-invariants using the simple complex $X(T A)$. In fact, by homotopy invariance of the $X$-complex for quasi-free algebras, one may even replace here $T A$ by any quasi-free extension $T$ of $A$.

For instance we get for the periodic cyclic homology of $A$ the formula

$$
H P_{*}(A)=H X_{*}(\hat{T})
$$

where $J \longmapsto T \rightarrow A$ is any extension of $A$ with $T$ quasi-free and $\hat{T}=\lim T / J^{n}$ is the $J$-adic completion. The ordinary cyclic homology groups $H C_{n} A$ can be determined from a natural filtration of $X(T)$ (a small modification of the $J$-adic filtration giving the same completion). The cohomology groups and more generally, the bivariant periodic cyclic homology groups for two algebras $A$ and $B$, are determined by the formula

$$
H P_{*}(A, B)=H_{*}(\operatorname{Hom}(X(\hat{T}), X(\hat{S}))
$$

where $S$ is a completed quasi-free extension of $B$ and Hom denotes the Hom-complex based on maps which are continuous for the adic topologies. From this definition we immediately get a product $H P_{i}(A, B) \times H P_{j}(B, C) \rightarrow H P_{i+j}(A, C)$. For the periodic cyclic theory this definition of the bivariant theory, given in [11] is better suited than the former definition of Jones-Kassel [17]. For instance any surjective algebra homomorphism $A \rightarrow B$ with nilpotent kernel induces an invertible element in $H P_{*}(A, B)$. However the inverse can not be realized as an element of the JonesKassel bivariant theory $H C^{*}(B, A)$. Also, excision holds for $H P_{*}$ (see section (7), but not for the Jones-Kassel theory.

One virtue of this new description of cyclic theory is that it reduces many computations to computations with the very simple $X$-complex for quasi-free algebras.

It has been shown in [10] that the fundamental properties of homotopy invariance and invariance [14] under nilpotent extensions, of the periodic theory, follow very naturally from this description. In fact, the invariance under nilpotent extensions is built in into the definition and homotopy invariance follows from a Cartan homotopy formula for the $X$-complex.

\section{MODELS FOR CYCLIC HOMOLOGY TYPES}

Motivated by the new description of cyclic homology in [11, Quillen in [28] went on to systematically compare the different ways that had been used in the literature to describe cyclic homology.

A mixed complex is a complex with differential $B$ and equipped with a second operator $B$ of degree +1 such that $[b, B]=B^{2}=0$. It is called free when its homology with respect to the differential $B$ is zero. 
An $S$-module is a complex with an operator $S$ of degree -2 commuting with the differential. It is called divisible when the operator $S$ is surjective.

A supercomplex is a $\mathbb{Z} / 2$-graded complex, i.e. a $\mathbb{Z} / 2$-graded vector space with an odd operator $d$ of square 0 . A tower of supercomplexes is an inverse system $X=X^{n}$ of supercomplexes indexed by the integers such that the maps $X^{n} \rightarrow X^{n-1}$ are surjective supercomplex maps. It is called special if the odd degree homology of the associated graded vanishes.

All mixed complexes, $S$-modules and towers of supercomplexes are assumed to be bounded below in the sense that the components $C^{n}$ or $X^{n}$ are zero for $n \ll 0$.

Let $\mathcal{C}_{\Lambda}, \mathcal{C}_{S}^{d}$ and $\mathfrak{T}^{s}$ denote the categories of mixed complexes, divisible $S$-modules and special towers, respectively.

There are natural comparison functors $\mathcal{C}_{\Lambda} \stackrel{\mathcal{B}}{\longrightarrow} \mathcal{C}_{S}^{d} \stackrel{\alpha}{\longrightarrow} \mathfrak{T}^{s}$. Moreover there are Hochschild, cyclic and periodic cyclic homology functors from each of these categories to vector spaces, and $\mathcal{B}, \alpha$ are compatible with those functors.

One of the essential results in [11] can be stated as saying that the special tower obtained from a natural filtration of $X(R)$ for a quasi-free extension $I \longmapsto R \rightarrow A$ of the algebra $\mathrm{A}$, is homotopy equivalent to the image under $\alpha \circ \mathcal{B}$ of the mixed complex $\Omega A$ with operators $b$ and $B$.

Quillen shows that the following five categories are equivalent in general:

(1) The derived category of mixed complexes.

(2) The homotopy category of free mixed complexes.

(3) The derived category of $S$-modules.

(4) The homotopy category of divisible $S$-modules.

(5) The homotopy category of special towers of supercomplexes.

The morphisms in each category can be viewed as cycles in a bivariant cyclic cohomology similar to the one of Jones-Kassel [17]. Each object in any of the categories describes a cyclic homology type in the sense that it contains the full information on the cyclic homology, Hochschild homology, negative cyclic homology and periodic cyclic homology. By the universal coefficient theorem established by Jones-Kassel for their bivariant theory, a cyclic homology type is determined, inside each of these categories, by its cyclic homology groups.

As a byproduct of these considerations, Quillen also relates the results in [1] to his earlier results in [27].

\section{EXCISION IN PERIODIC CYCLIC HOMOLOGY}

The results in [11] had established a very satisfactory framework for cyclic theory explaining smoothly some fundamental properties such as homotopy invariance, Morita invariance, invariance under nilpotent extensions and furnishing a natural picture for the Chern character maps from $K$-theory to cyclic homology. Especially in the periodic case the bivariant theory thus now looked formally exactly like the equally $\mathbb{Z} / 2$-graded bivariant topological $K$-theory of Kasparov which plays a fundamental role in noncommutative geometry. It became completely clear that the missing ingredient to relate $K$-theory and cyclic theory was excision - the fact that 
any extension of algebras induces long exact sequences in the two variables of the bifunctor.

The starting point for our attack on the excision problem in periodic cyclic theory was the observation in [8, 9] that if $I$ is an ideal in a quasi-free algebra $R$, then the projective system $\left(I^{n}\right)$ of powers of $I$ satisfies a property which is analogous to Wodzicki's $H$-unitality (namely the property that the multiplication map $I \otimes I \rightarrow I^{2}$ admits an $I$-linear splitting). Let $C(A)$ be the complex describing periodic cyclic cohomology $H P^{*} A$ of an algebra $A$ and consider an extension of algebras of the form $0 \rightarrow I \rightarrow P \rightarrow Q \rightarrow 0$. We may then take the inductive limit over $n$ for the extensions of complexes

$$
0 \rightarrow C\left(I^{n}: P\right) \rightarrow C(P) \stackrel{\pi}{\longrightarrow} C\left(Q / I^{n}\right) \rightarrow 0
$$

where $C\left(I^{n}: P\right)$ denotes the relative complex, i.e. the kernel of $\pi$. An argument à la Wodzicki shows that the cohomology of the first complex will converge in the inductive limit to $H P^{*}(I)$ while, by invariance of $H P^{*}$ under nilpotent extensions [14], the cohomology of the third complex will converge to $H P^{*}(Q)$. The limit of the associated long exact cohomology sequence for this exact sequence of complexes will then be of the form

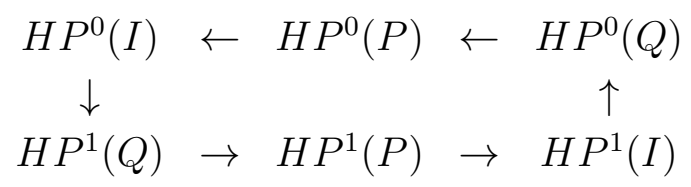

In a second step it was noted in 9, that every extension $0 \rightarrow I \rightarrow A \rightarrow B \rightarrow 0$ can be mimicked by an equivalent extension $0 \rightarrow I^{\prime} \rightarrow A^{\prime} \rightarrow B^{\prime} \rightarrow 0$ where $I^{\prime}$ is isomorphic to an ideal in a quasi-free algebra. This established excision for periodic cyclic cohomology in general. The excision problem for periodic cyclic homology remained open at that point.

It is possible, even though not trivial, to derive from the excision result for periodic cyclic cohomology the one for periodic cyclic homology, using homological algebra. A problem in doing so is the more delicate behaviour of homology and exact sequences under inverse limits rather than direct limits. However, such a computational approach is not good enough. For one thing, it would not work for topological algebras, but, more importantly, it would not give the result for the bivariant theory. The excision result for the bivariant theory is very important, even if one is only interested in the monovariant theory. For instance, the connecting maps in the long exact sequences for periodic cyclic homology and cohomology are given by the product by a natural bivariant element determined by the extension. The method to attack that technical problem, that was used in [13], was to cast the entire theory into the framework of pro-vector spaces, pro-complexes and at the same time to extend it from algebras to pro-algebras (by definition a pro-object is an inverse system in a category). An important feature of the category of pro-vector spaces is the fact that it contains enough projective objects. On the other hand, if $0 \rightarrow I \rightarrow R \rightarrow A \rightarrow 0$ is a quasi-free extension, then the pro-complex $X\left(R / I^{n}\right)$ which describes the periodic theory of $A$ can be replaced by the pro-complex $X\left(I^{n}\right)$ 
which describes the same theory (up to a dimension shift even $\leftrightarrow$ odd). However this second complex is a complex of projective pro-vector spaces. $\operatorname{Thus} \operatorname{Hom}\left(X\left(I^{n}\right), \cdot\right)$ is exact on pro-vector spaces.

Using this technology, the argument for excision in periodic cyclic cohomology outlined in [8] carries over to the bivariant theory - but in a more conceptual way, since inductive and projective limits are already incorporated into the notion of a morphism between pro-vector spaces.

Theorem 7.1. [13] Let $0 \rightarrow S \rightarrow P \rightarrow Q \rightarrow 0$ be an extension of algebras and $A$ an algebra. There are two natural six-term exact sequences

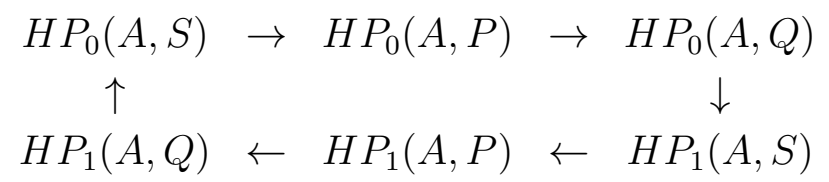

and

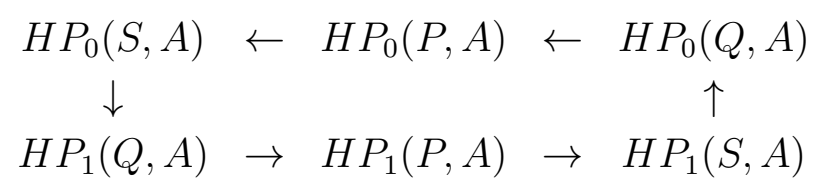

where the horizontal arrows are induced by the maps in the given extension and the vertical maps are given by the product with a natural class in $H P_{1}(Q, S)$, associated with the extension.

A simplified proof of this theorem - even more directly in the spirit of the framework of [11] and avoiding Wodzicki's argument completely - was given later by R.Meyer [20]. Let $S \longmapsto P \rightarrow Q$ be an extension. The basic idea of Meyer's proof is to use the left ideal $L \subseteq T P$ that is generated by $S \subseteq T P$. Meyer shows that $L$ is quasi-free and that the complex $X(L)$ is homotopy equivalent to the kernel of the canonical map $X(T P) \rightarrow X(T Q)$. Secondly, he shows that $X(L)$ is homotopy equivalent to the kernel of the canonical map $X(T P) \rightarrow X(T Q)$. The excision theorem follows.

\section{LATER DEVELOPMENTS IN CYCLIC THEORY}

The approach to cyclic theory developed in [10], [11], [13] turned out to be a very natural and convenient basis for many further advancements in cyclic theory.

The author developed a bivariant $K$-theory for locally convex algebras and used the excision result of [13] to construct a bivariant Chern-Connes character from this bivariant theory to bivariant periodic cyclic theory.

Connes' entire cyclic cohomology [3] was generalized by Meyer, using the framework of [11, to the bivariant setting and to bornological algebras, see [21]. Meyer also proved excision for this theory.

M.Puschnigg developed the very flexible local theory which works for $C^{*}$-algebras just as well as for locally convex dense subalgebras [23], 24]. He obtained the important result that the local theory of a $C^{*}$-algebra is independent of the choice of a natural dense "smooth" subalgebra and constructed a bivariant Chern-Connes character from Kasparov's $K K(A, B)$, for $C^{*}$-algebras $A, B$, to his $H C^{l o c}(A, B)$. 
C.Voigt extended equivariant periodic theory which was known before only for compact group actions and only for homology [2, [1], to actions of general groups and to cyclic cohomology and to the bivariant theory. A crucial point was again the Homcomplex for $X$-complexes (which are not quite complexes in this case) for quasi-free extensions [31], 30].

\section{REFERENCES}

[1] Jonathan Block and Ezra Getzler. Equivariant cyclic homology and equivariant differential forms. Ann. Sci. École Norm. Sup. (4), 27(4):493-527, 1994.

[2] Jean-Luc Brylinski. Algebras associated with group actions and their homology. preprint, Brown University, 1987.

[3] A. Connes. Entire cyclic cohomology of Banach algebras and characters of $\theta$-summable Fredholm modules. K-Theory, 1(6):519-548, 1988.

[4] A. Connes and J. Cuntz. Quasi homomorphismes, cohomologie cyclique et positivité. Comm. Math. Phys., 114(3):515-526, 1988.

[5] Alain Connes. Noncommutative differential geometry. Inst. Hautes Études Sci. Publ. Math., (62):257-360, 1985.

[6] Joachim Cuntz. Universal extensions and cyclic cohomology. C. R. Acad. Sci. Paris Sér. I Math., 309(1):5-8, 1989.

[7] Joachim Cuntz. Cyclic theory, bivariant $K$-theory and the bivariant Chern-Connes character. In Cyclic homology in non-commutative geometry, volume 121 of Encyclopaedia Math. Sci., pages 1-71. Springer, Berlin, 2004.

[8] Joachim Cuntz and Daniel Quillen. On excision in periodic cyclic cohomology. C. R. Acad. Sci. Paris Sér. I Math., 317(10):917-922, 1993.

[9] Joachim Cuntz and Daniel Quillen. On excision in periodic cyclic cohomology. II. The general case. C. R. Acad. Sci. Paris Sér. I Math., 318(1):11-12, 1994.

[10] Joachim Cuntz and Daniel Quillen. Algebra extensions and nonsingularity. J. Amer. Math. Soc., 8(2):251-289, 1995.

[11] Joachim Cuntz and Daniel Quillen. Cyclic homology and nonsingularity. J. Amer. Math. Soc., 8(2):373-442, 1995.

[12] Joachim Cuntz and Daniel Quillen. Operators on noncommutative differential forms and cyclic homology. In Geometry, topology, $\mathscr{E}$ physics, Conf. Proc. Lecture Notes Geom. Topology, IV, pages 77-111. Int. Press, Cambridge, MA, 1995.

[13] Joachim Cuntz and Daniel Quillen. Excision in bivariant periodic cyclic cohomology. Invent. Math., 127(1):67-98, 1997.

[14] Thomas G. Goodwillie. Cyclic homology, derivations, and the free loopspace. Topology, 24(2):187-215, 1985.

[15] Nigel Higson. The residue index theorem of Connes and Moscovici. In Surveys in noncommutative geometry, volume 6 of Clay Math. Proc., pages 71-126. Amer. Math. Soc., Providence, RI, 2006.

[16] Arthur Jaffe, Andrzej Lesniewski, and Konrad Osterwalder. Quantum $K$-theory. I. The Chern character. Comm. Math. Phys., 118(1):1-14, 1988.

[17] John D. S. Jones and Christian Kassel. Bivariant cyclic theory. K-Theory, 3(4):339-365, 1989.

[18] Maxim Kontsevich and Alexander L. Rosenberg. Noncommutative smooth spaces. In The Gelfand Mathematical Seminars, 1996-1999, Gelfand Math. Sem., pages 85-108. Birkhäuser Boston, Boston, MA, 2000.

[19] Jean-Louis Loday and Daniel Quillen. Cyclic homology and the Lie algebra homology of matrices. Comment. Math. Helv., 59(4):569-591, 1984.

[20] Ralf Meyer. Excision in entire cyclic cohomology. J. Eur. Math. Soc. (JEMS), 3(3):269-286, 2001. 
[21] Ralf Meyer. Local and analytic cyclic homology, volume 3 of EMS Tracts in Mathematics. European Mathematical Society (EMS), Zürich, 2007.

[22] Denis Perrot. A bivariant Chern character for families of spectral triples. Comm. Math. Phys., 231(1):45-95, 2002.

[23] Michael Puschnigg. Excision in cyclic homology theories. Invent. Math., 143(2):249-323, 2001.

[24] Michael Puschnigg. Diffeotopy functors of ind-algebras and local cyclic cohomology. Doc. Math., 8:143-245 (electronic), 2003.

[25] D. Quillen. Chern-Simons forms and cyclic cohomology. In The interface of mathematics and particle physics (Oxford, 1988), volume 24 of Inst. Math. Appl. Conf. Ser. New Ser., pages 117-134. Oxford Univ. Press, New York, 1990.

[26] Daniel Quillen. Algebra cochains and cyclic cohomology. Inst. Hautes Études Sci. Publ. Math., (68):139-174 (1989), 1988.

[27] Daniel Quillen. Cyclic cohomology and algebra extensions. K-Theory, 3(3):205-246, 1989.

[28] Daniel Quillen. Bivariant cyclic cohomology and models for cyclic homology types. J. Pure Appl. Algebra, 101(1):1-33, 1995.

[29] William F. Schelter. Smooth algebras. J. Algebra, 103(2):677-685, 1986.

[30] Christian Voigt. Equivariant local cyclic homology and the equivariant Chern-Connes character. Doc. Math., 12:313-359 (electronic), 2007.

[31] Christian Voigt. Equivariant periodic cyclic homology. J. Inst. Math. Jussieu, 6(4):689-763, 2007.

[32] Mariusz Wodzicki. Excision in cyclic homology and in rational algebraic $K$-theory. Ann. of Math. (2), 129(3):591-639, 1989.

Mathematisches Institut, Einsteinstr.62, 48149 MÜnster, Germany

E-mail address: cuntz@uni-muenster.de 